\title{
Systematizing benefits of open science practices
}

\author{
Valeria Arza ${ }^{\mathrm{a}, \mathrm{b}, *}$ and Mariano Fressoli ${ }^{\mathrm{a}, \mathrm{b}}$ \\ ${ }^{\text {a }}$ Research Center for the Transformation (CENIT), Argentina \\ ${ }^{\mathrm{b}}$ National Scientific and Technical Research Council (CONICET), Argentina
}

\begin{abstract}
Open science aims at the creation of public scientific goods by means of sharing outputs and widening and facilitating collaboration, in one or many of the different research stages. There are many beneficial aspects of open science that have been claimed in the literature, such us improving research efficiency, accelerating creativity, democratizing knowledge and empowering stakeholders. These claims are normally based on anecdotal experiences. In this paper we aim at organizing the extant literature on benefits of open science, in an attempt to build a bi-dimensional framework that relates characteristics of openness with benefits to be expected. The first dimension accounts for the characteristics of the collaboration, while the second for aspects of access to shared outputs. In the conclusion, we briefly illustrate our framework using evidence from four Argentinean open science initiatives.
\end{abstract}

Keywords: Open science, analytical framework, research, science policy

\section{Introduction}

In modern scientific tradition, collaboration among scientists and the production of scientific public goods have been the engine for scientific production and the justification for public investment in science [18]. Scientists have been expected to collaborate across disciplines and over generations so as to contribute to a stock of interconnected knowledge needed for scientific advance. This knowledge would be publicly shared and disseminated through publications [40]. However, in practice, scientific knowledge production has been much more closed, fragmented and isolated from social problems than the idealist conception of modern science expected, as a result of three phenomena:

Firstly, scientific practice has become locked in the pursuit of personal/individual success. Scientists compete to reach priority and much of their knowledge is not transmitted. This is due to fear of competition, criticism, convention in a given field or the intrinsic characteristics of the tacit knowledge involved. Thus, although scientists publish their results, some of the relevant information to be able to construct knowledge cumulatively is not published [24]. Notoriously, negative results of experiments are not generally published. As a result, scientific production has been much less collaborative than it could have been and also less transparent. Resources become misused affecting negatively research productivity and reproducibility (and therefore reliability).

Secondly, assessment schemes have been increasingly influenced by marketing strategies of academic publishers, which push for the use of quantitative indicators based on citations as proxy for research

\footnotetext{
*Corresponding author. Tel.: (5411) 4373-3714; E-mail: varza@ fund-cenit.org.ar.
} 
quality. Thus, researchers worldwide are motivated to guide their research to areas, topics and methods that would be widely cited worldwide [31], which does not need to coincide with societal needs [33].

Thirdly, scientific policies oriented to the commercialization of scientific knowledge have increasingly locked up scientific knowledge. Political pressures in the developed world have urged scientific production to demonstrate its social and economic utility [43,44]. In turn, intellectual property mechanisms implied the protection of scientific knowledge that previously remained in the public domain [16,39], so as to motivate private sector to invest in scientific production. These practices accelerated the processes of occlusion of science; knowledge become protected and could only be used with the owner authorization, with two different effects. Firstly, scientific incentives drove scientific production away from the idea that knowledge is a public good. Secondly, the virtue of learning collaboratively and the collective creation of cumulative knowledge stocks as platforms for future knowledge production became seriously endangered, affecting the rate of invention.

In parallel to these developments, the emergence and wide diffusion of ICTs created ever increasing opportunities for sharing and collaboration, which shortened geographic, disciplinary and expertise distances. There exist various technologies, tools and infrastructure that facilitate collaborative production processes in various social spheres, and scientific production is not and exception.

These new opportunities extended the boundaries of what is feasible to share and how to do it, enlarging the potential scale and scope of collaboration and openness in science $[24,26]$. For example, other resources besides publication can now be shared; such as data, lab notes, infrastructure, etc. ICTs also broadened the range of actors and expanded the possible time for collaboration; the contributions can be brief and there are tools to improve accessibility to facilitate the collaboration of actors with different backgrounds. Similarly, ICTs also broadened the range of actors and extended the possible time of collaboration, and the contributions can be brief and there are tools that facilitate the collaboration of actors with different capacities and expertise. In addition, new technologies such as big data, machine learning, massive use of sensors, drones and greater availability of low-cost scientific tools are changing the way knowledge is produced.

The experience of open source software created an important precedent for the open science movements in terms of know-how and visions. Open software become a community of practice where open access to knowledge and wider collaboration overcome the old prejudices that only competition allocates resources efficiently. Open source software demonstrated for several years now that massive and open collaboration works, and that it could even become mainstream practice in fields where information is a key input [57]. In fact, current open-minded movement, in science and other fields, is inspired by free software and open source activists. ${ }^{1}$ They probed that sharing sums up.

Open science is rapidly changing how science is being produced and used. However, as with other buzzwords and fashion terms, there is no single definition of open science. There are different understandings, motivations and potential benefits from open science $[21,28]$.

However, they all aim at (i) producing public goods: publications, data, infrastructure, and tools available to all; (ii) encouraging greater collaboration among scientists from different disciplines and academic fields; and (iii) broadening the diversity of science-producing actors. By these means, efficiency in scientific production is enhanced, scientific knowledge is democratized and science becomes better connected with societal needs. These potential benefits work as motivational goals for the different

\footnotetext{
${ }^{1}$ Efforts to apply open source ideas to science can be traced back to the late 90 s and early 2000 s. These include several declarations in favor of open access including the Budapest, Bethesda and Berlin declarations. The role of new creative commons licenses was also important in order to allow scientist to manage their publications. Finally, there were direct efforts from people closed to Creative Commons to create initiatives around scientific commons [50].
} 
meanings and practices of open science. However, there is yet little understanding on mechanisms and conditions that link open science practices with potential benefits. There is no guarantee that opening up some scientific practices or outputs in some way would univocally trigger knowledge democratization, research efficiency, and social responsiveness.

This paper aims at disentangle different meanings for open science and organize them so as to relate them with claims on benefits as referred in the literature. We argue that the wide array of open science practices could be displayed in a two dimensional space, with one dimension being features of collaboration in processes and the other being the characteristics of access to outcomes. The specific location in this space anticipates different types of expected benefits. Our contention is that this analytical framework could be used as a toolbox to assess different experiences of open science around the world against their proposed goals.

The paper is organized as followed: Section 2 discusses benefits of open science as informed by the literature; Section 3 organizes those benefits in Cartesian diagram with collaboration and access as main dimensions to characterize open science practices; Section 4 concludes by illuminating our framework using case-study evidence from Argentina.

\section{Benefits associated to open science as claimed by the literature}

Different strands of the scatter literature analyzing open science practices claim they trigger several benefits, which we organized in three groups:

\section{i. Improving scientific efficiency}

One of strong argument for supporting open science practices is that they increase efficiency [45]. ${ }^{2}$ This is the result two mechanisms: a) wider availability of knowledge resources that makes research cheaper and research success more likely and b) more fluent collaboration among heterogeneous knowledge actors that amplifies collective intelligence and creativity.

Open access to scientific final or intermediate outcomes, increases the pool of knowledge in common use. This increases efficiency because unnecessary duplication can be more easily avoided and because researchers can explore new questions and solutions to problems by standing on the shoulders of a taller giant. Sharing promotes beneficial spillovers among research programs and makes the most of investment in science [17]. Moreover, open access also increases efficiency because it enables the use of computing power machine that interconnecting everything that is already known, reusing online available data to arrive to new findings. This new capacity has been sometimes name as data driven intelligence [45], and depends on open access to use automated tools to mine the literature. In turn, open data allows reproducibility of key research findings (and also experimental methods) that could push science ahead [29].

However, it is not just availability of publication and data that helps. Digital tools have also opened up opportunities for a greater quantity of actors from a wider community, not just professional scientists, to participate directly in scientific production overcoming restrictions imposed by physical and cognitive distance [60]. Sometimes they participate in data collection (see Galaxy Zoo, Foldit and Great Sunflower Project) proving the scientific endeavor with new cognitive and manpower resources $[24,45]$.

\footnotetext{
${ }^{2}$ To increase efficiency in scientific production means to be able to achieve more or better scientific outputs (i.e. findings, publications, trained scientists) using the same amount of scientific inputs (i.e. resources). This relates to costs advantages or to learning advantages of openness and collaboration. In turn, we may refer also to dynamic efficiency when there is an increase in the likelihood of improving efficiency in the future given current state of the art.
} 
In turn, collaboration and interaction with the community improves efficiency also by boosting creativity. Open science practices sometimes involve communities participating in analytical or design research stages (not just data collection). In those cases, non-academic actors or scientists from different disciplines could contribute by drawing knowledge resources and cognitive tools from their own experience, which throw new light to research problems. Social studies of science claimed that major innovation in different fields tend to be put forward by scientists trained in different disciplines, mainly because they are not bound by professional traditions [8]. A similar phenomenon has been observed in studies about innovation [12]. Jeppensen and Lakhani (2010) [32] claim that it is not just technical marginality but also social-political marginality which may contribute with novel ideas, for similar reasons, these actors are more prone to thinking unconventionally and therefore more creative. ${ }^{3}$ Wider participation and interaction among diverse set of actors enable the mechanism known as 'the wisdom of the crowds' [45,55], which basically states that a group could better solve a problem than any single individual from the same group.

Finally, collaboration among scientists in the same field triggers a different mechanism to improve efficiency. When they are able to interact fluently, collective intelligence is amplified by the mere fact of being able to share, validate and quickly rule out different ideas, assumptions, hypotheses or avenues of inquiry [45]. ${ }^{4}$ This consequence of collaboration is greater when using web technologies because it gets across once unconceivable distances of time and space and ideas could quickly go back and forward feeding from the interaction, augmenting the capacity to solve problems (see, for example, the Polymath project).

\section{ii. Improving democratization of scientific knowledge}

There are three complementary mechanisms through which open science practices democratize scientific knowledge: by improving access to scientific resources; by enabling the participation of a wider community in the research process; and by making science better understandable for a wider population.

Open access movements emerged as a reaction to the closure of scientific knowledge imposed behind paywall to access scientific publications. While the rate of scientific production has been always increasing, the distribution in the possibility of using such knowledge has remained unequal [15]. Aronson (2004) [4] estimated that $56 \%$ of institutions in lowest income countries have no subscriptions to international journals in medical research. Open access is potentially democratising because it reduces the costs of using and reusing the worldwide accumulation of knowledge.

Open access increases the pool of information available to anyone not just scientists. Nurses, patients, teachers, students may get to interested to learn about latest treatment of certain diseases; small businesses may get to know about relevant techniques in several application fields; etc. A recent survey to Latin American users of open access portals show that $25.2 \%$ of articles were downloaded for non-academic use; either to satisfy personal interests $(10.5 \%)$ or for professional practice unrelated to scientific production (non for profit: $4.2 \%$, private: $3.8 \%$, public $6.7 \%)^{5}[1]$.

\footnotetext{
${ }^{3}$ However, greater collaboration with non-scientific actors will probably require a lot of boundary work to translate scientific information to a wider public (see [36]).

${ }^{4}$ Nielsen (2012) [45] argues that such amplification of collective intelligence probably works better when interactive actors share at least some cultures of practice or when they are focused on the same problem-solving strategy.

${ }^{5}$ Data corresponds to 58957 downloads from Scielo. The author also analyzed 22910 downloads from Redalyc and in that case there was $16 \%$ downloads for non-academic use, split into personal interests (7.9\%) and professional non for profit $(2.9 \%)$; private $(1.9 \%)$; and public $(3.4 \%)$.
} 
The same could happen with open data; when properly curated and easily available, it could be used by different actors including scientist from different disciplines but also the non-scientific actors such as NGOs, firms, and just citizens (see for instance [34]).

Scientific publications and data are an outcome of research which is largely funded with public investment (see [46,54]). Thus, it is just fair that everyone could access to the outcome of the efforts of everyone. This idea is so powerful, that open access to data and publications as a way to improve the actual use of scientific knowledge, has become the focus of several public policies initiatives promoting open science. ${ }^{6}$

Open access contributes to a better informed society and fosters new processes of learning [27,56,61], which drives us to the second claim on open science as a democratizing force. Some open science practices promote wider participation of the society in the production of scientific knowledge. One example is citizen science projects, in which non-academic actors contribute to the production of scientific knowledge in disciplines like ornithology, astronomy and environmental conservation [14]. The emergence of new digital tools and web based protocols for gathering data is widening the scope of people that can participate of scientific research beyond "a privileged few" [51]. Furthermore, participation in the production of scientific data allow learning processes leading to the construction of new questions and skills and, eventually, the development of forms of "science by the people" (see [37]). In cases such as biohackers and do-it-yourself data recollection projects, this has challenged the hierarchies and traditional orientation of science (see [19]).

However, there are still costs associating to training potential users so they become able to enjoy all functions of shared outputs and make the most of open access. These costs are inversely related to the investment in knowledge translation and communication efforts, and as Catlin-Groves (2012) [14] suggested, more complex data involvement from non-scientific actors will demand more training. This point links to the third motivation for open science projects associated to democratization: to make science understandable for a wider public [21] by fostering scientific education [60] or by designing tools and exploring new channels to disseminate scientific information (see [13]).

There is a multiplicity of approaches to the dissemination of science [7]. Traditionally, the focus was on closing the information gap regarding scientific knowledge. In the mid-1980s, public understanding of science emerged, seeking to raise the level of scientific knowledge in the public to reverse the growing distrust of scientific expertise. In the same vein, more recently, new outreach trends have emerged, based on the use of interactive techniques (games, videos, experiments, etc.) to encourage learning during practice rather than passive information consumption [23]. According to Wiggins and Crowston (2011) [60], several open science projects can be considered as educational projects that offer formal and informal learning services. There are also other initiatives promoting scientific education directly, such as online forums and online training courses such (tutorials, massive online courses, etc.) (see for instance [41]). Some open science initiatives are starting to introduce open science tools in students curricula as a way to improve learning and research capabilities [6].

\footnotetext{
${ }^{6}$ This includes, for example, the implementation of norms that commit scientists to make their publications and data freely available; changes in the form of the evaluation acknowledging and incentivizing the publication of the datasets [47,53]; the creation of open digital repositories; the promotion of learning in management and data analysis [20]; the creation of incentives and mechanisms of acknowledging the support of the development of an open (software and tools) infrastructure [48,53]; and the generation of new forms of publicly communicating science [20]. In Latin America Argentina and Perú are pioneer countries to get specific legislation to guarantee open access to publicly funded scientific outputs. In Argentina open access policies are institutionalized by the enactment of The National Law for the Creation of Digital, Institutional and Open Access Repositories (approved in 2013 and fully in force since 2016). After Argentina and Peru, other countries in the region started to move along similar paths.
} 


\section{iii. Improving research capacity to attend societal needs}

There are three mechanisms claimed by the literature on how open science practices improve the research capacity to solve societal needs.

Firstly, wider access helps visibility. Open science practices could help local problems to become visible and better communicated [53]. When using digital tools and social networks the dissemination of open access information allows that problems affecting powerless actors to become better known [56]. Marginalized groups could become better endowed with knowledge resources and political support to engage negotiation with other actors like authorities, the press or other potential supporters that could contribute to solving their problems [15].

Secondly, by promoting community actors to participate in the scientific endeavor the research agenda could be better guided towards solving problems affecting that group [20,53]. Moreover, when the community gets involved in research, people could grab from their own informed experience to offer inputs for developing solutions, improving therefore the final outcome.

Finally, the open availability of scientific resources deters private appropriation of such resources. This could contribute to find cheaper solutions to societal problems. Open access and open licenses such as creative commons avoid the creation of barriers that hamper the process of turning scientific knowledge into concrete solutions to local problems. The societal impact of scientific research depends, in turn, on the potential for promoting a wide appropriation of research outcomes, through open access and open licenses [38]. This reacts against the phenomenon known as the "tragedy of the anti-commons", which turns out when there is such an accumulation of patents on small fractions of knowledge that makes it cumbersome and highly costly to combine all of those separate elements to produce useful solutions [30]. In contrast, open science practices are then seen as an alternative business model that could solve the anti-commons problem relying on open access, worldwide collaboration and open licenses. An interesting area where there is experimentation is open source drug discovery. These projects are creating open knowledge resources that could be freely used (e.g. Open Source Malaria [49]; Open Source Drug Discovery [2,11]; Malaria Box [52,58]; among others). Most of them, and not by chance, are oriented to produce drugs for tropical disease, where the economic rewards are low and not enough for large companies to get into business.

\section{Conceptual framework to organize open science practices}

This section aims at organizing the different meanings of open science in an attempt to better relate practices with potential benefits. The intention is not to create an ideal type of open science, but rather to visualize some common aspects and, at the same time, to highlight that there are different paths to improve efficiency, democratization, and societal responsiveness of scientific practice. Open science practices have been previously classified according to: i) what is shared (e.g. publication, data tools etc.); ii) how it is shared and, iii) with whom to share [48]. We build on this classification to create a bi-dimensional framework for open science practices.

We use Benkler's twofold characterization of open and collaborative knowledge production $[9,10]$. A first dimension characterizes how actors collaborate among each other to produce knowledge, and a second one characterizes access to shared outcomes. Thus, while the first dimension characterizes social exchange of ideas to produce knowledge, the second one refers to existing institutions that regulate the capacity of social actors to use knowledge resources. 
There are different aspects of collaboration that matter to achieve beneficial outcomes. We claim the scale of participation is important to activate mechanisms such as 'the wisdoms of the crowds', or the 'collective intelligence', or to reduce the costs of producing research as in the collection of data in citizen science practices. We also argue that not just scale matters but also the level of interaction among participants. Process of collective intelligence, for example, will not occur iF participants do not have the chance to rapidly rule out or validate their ideas [45]. In addition, learning is always an interactive process [35] and learning is key for democratization. Moreover, also diversity or a participation of a wider community in the scientific endeavor matters for the democratization of science, and for other mechanisms related to efficiency such as 'the wisdoms of crowds'. Finally, another aspect related to collaboration that matters especially for societal responsiveness but also for democratization is the degree of participation and commitment [3].

The second dimension aims, in turn, to take into consideration aspects of access to shared resources. This is related to the common based characteristics of shared resources. As in open source, the backbone principle of open science practices is that scientific resources should be used and re-used by everyone. However, there are formal and informal restrictions that make this principle work to different extent in practice. For example, open access could be restricted by different types of paywalls (e.g. subscriptions to journals or licenses to used patented knowledge) or other formal restrictions to use, distribute, reproduce, etc. [42]. There may be also informal restrictions to use and re-use knowledge resources related to the specific skills, capabilities or capital resources needed for using shared scientific outputs.

When relating this dimension of open science with potential benefits, we could realize that some specific aspects of access matters relatively more in some cases than others. Unrestricted open access to publications and data matters for mechanisms affecting efficiency, such as 'data-driven efficiency'. For achieving these benefits, it would be enough to guarantee open access to academic actors. However, for democratization open access is needed also for a wider community. In turn, what really matters for democratization is to improve the accessibility to scientific knowledge to guarantee that a larger quantity and wider variety of actors become endowed with knowledge resources. Improving the communication of science could help in this case. Similarly, for solving societal needs accessibility is needed but what becomes crucial is to augment the visibility of societal needs and achievements [22]. For that aim, no just communication techniques but also a diversification of channels of communication could help.

These bi-dimensional characteristics of openness and collaboration could be drawn in a Cartesian diagram such as that in Fig. 1. We also include in the Figure the different mechanisms and the associated potential benefits as have been discussed above. The actual location of benefits in the Figure is speculative. It was done by imagining that each of the different aspects of collaboration and access pulls towards the vertical or the horizontal end respectively. Thus, for efficiency, we venture that the collaboration dimension is particularly important, while both of them are important for societal responsiveness but especially for the democratization of science.

\section{Conclusions}

This paper organizes different elements of openness in order to relate them to specific benefits claimed by the open science literature. We argue that benefits are related to the specific characteristics of the opening process. We built an analytical framework based on eight aspects (Scale, Diversity, Interaction, Participation, Visibility, Accessibility, Access by scientists and Access by everyone) of two key dimensions of open science: collaboration and access. 


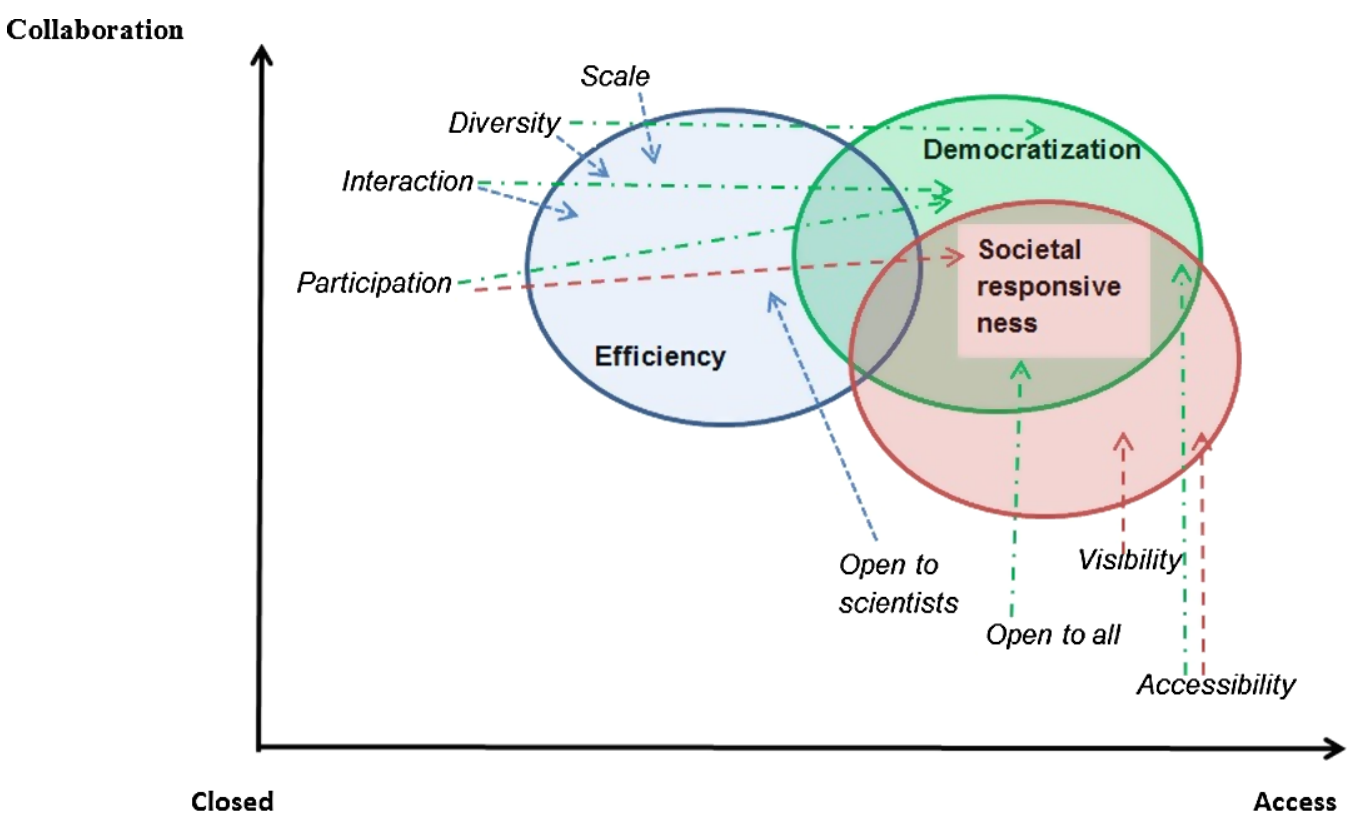

Fig. 1. Two dimensions of open science.

In previous studies [25] and [5] we produced case study evidence on open science projects in Argentina. Some of the open science projects we studied were:

- New Argentinean Virtual Observatory - NOVA (astronomy): Researchers from various institutions in the country became organized to create a digital platform that aimed at storing and sharing already processed astronomical data. It facilitates collaboration of local and international astronomical community, through documentation, digitization and open access to data.

- Argentinean Project of Monitoring and Prospecting the Aquatic Environment - PAMPA2 (limnology): it is an interdisciplinary network of scientists from seven different research laboratories, that seek to understand the reaction and behavior of water from lakes to improve the design of management plans that may prevent deterioration and to preserve the populations' health. They monitor several lagoons with buoys equipped with automatic sensors capable of measuring temperature, pressure, wind, rainfall, etc. These data are transmitted in real time to the laboratories responsible for its operation and can be openly accessed for their website.

- e-Bird Argentina (ornithology): is a citizen science project that receives and gathers in an online platform, bird sightings from anybody in any part of the world. The platform is open access and it aims at managing and sharing online data of bird sightings made by amateur and professional watchers. eBird makes use of free software tools and online collaboration to efficiently gather, archive, and distribute information about birds to a much wider audience. The online platform was developed in the United States in 2002 by the Ornithology Laboratory at Cornell University and the National Audubon Society. In Argentina the portal started in 2013.

- Integral Management of the Territory - IT (geography-chemistry): An interdisciplinary group of researchers designed an action-research project for integrated land management seeking to relieve the needs of two particularly affected areas by floods in 2013. Neighbors from these areas actively participated in project by designing the survey to be used to gather data (their views and needs were 
included from the beginning in the questionnaire), and in the sampling of rainwater, which measure their level Ph.

Using data from these case studies, we could relate the specific features of openness and collaboration with three reported benefits of open science as discussed in the literature: efficiency, democratization and social responsiveness.

The four initiatives are heterogeneous in terms of dimensions of openness. Some aim at increasing collaboration while others are mostly based on improving access, accessibility and visibility of scientific outcomes. There are several directions of openness and, as a result, potential benefits also differ.

From the four cases eBird is the case that ranks high in most dimensions, which drives to high levels of expected efficiency, democratization and social responsiveness. In fact, eBird's platform enabled the interaction among professionals and birdwatchers around the country that improved the quantity and quality of stock of shared resources and allowed the generation of a large database, updated daily. Moreover, publications by Argentinean eBird representatives have doubled since the beginning of the project, while the annual citations to their work have more than tripled. Something similar can be said about the potential for democratization and social responsiveness. Data is open access, the platform is very user-friendly, they advertise vastly their initiative (party relying on international efforts in this regard) and the infrastructure is open source.

NOVA has been very beneficial in terms of data sharing and data re-use among astronomers. The project has done a great effort to take astronomic data and images out from individual computers and to share them openly with everyone. This has improved the quantity of information that is available for common use. Since the initiative started in 2009, there has been 125.075 data downloads. As in the case of eBird, the project leader has dramatically increased the number of annual publications and their annual citations (128\% and 332\% increase, respectively). The other important expected benefit is democratization. Although our evidence suggests that NOVA ran a bit short in terms of amplifying its impact beyond the scientific community (e.g. their platform is not very accessible for the wide public), this has been changing lately with the creation of sister project which uses citizen science practices (Galaxy conqueror). This has improved the diffusion of astronomy among the wide public, and it might also contribute to capacity building and democratization of science, as has been observed in similar cases such as Galaxy Zoo.

Something similar happens with PAMPA2. Expected benefits seem to be primarily related to democratization. Evidence suggests that this is very much related to its international spin off project (SAFER), which is trend-setting in the use of community based strategies to produce knowledge and to manage natural resources. PAMPA2 does seem to be doing quite well in terms of scientific performance. The group managed to create an interdisciplinary network of scientists who collaborate locally and internationally. Actually, open access to data has opened up opportunities to participate in new international projects widening local scientists' networks. The evolution of annual publications and citations has increased in $218 \%$ and $144 \%$ respectively since the beginning of the project.

Finally, although the topic being investigated is central for communities, social responsiveness does not seem to be one of the promises of PAMPA2. It does not experiment with citizen science tools for data collection and it does not have a community capability building component.

In turn, the main expected benefit for IT is social responsiveness. The project was an ongoing project at the time of our case study, so we cannot really assess its benefits. The local community that participated in the project has increased their knowledge about territorial planning and they have also collected some data that could back their claims in the future. Thus, it does seem to be some evidence that the project is oriented towards achieving this expected benefit. Regarding democratization, although the diversity 
and commitment to citizen participation lead us to expect knowledge democratization this achievement was somehow hindered by the political context in which the project emerged. Researchers said that it was puzzling to work with local communities in the context of political disputes (with local authorities), because they (the researchers) did not want to create false expectations on the outcomes of the project, while at the same time they needed to motivate the community to be part and committed to it. One specific and important problem faced by the project at the time of the interviews was political barriers to enable open access to data. Local authorities retained the right to decide when it was a reasonable (political) time to show certain results and to define what and when solutions would be carried out. Efficiency, in turn, does not seem to be one of the main promises in terms of how the imitative was designed and in fact, our interviews showed multidisciplinariety somehow risked the likelihood of obtaining publishable outcomes, partly because specialized journals normally belonged to certain disciplines and also because the final outcomes depended on the commitment of other researchers in a context where quality could not be cross-checked due to lack of specific skills. Annual publications and citations have increased annually since 2013, but much more moderately in comparison to the other mentioned initiatives (56\% and 33\%).

In sum, our conceptual framework helped us to identify the main expected benefits of each initiative which were largely validated by evidence we collected for each of the case studies.

The implications of these finding are that there is no need to commit to total openness to enjoy benefits of open science. There is no one single pathway to opening up; there are diverse dimensions scientists could explore, depending on their goals. Actually, in line with Whyte and Pryor (2011) [59] our findings show that researchers do not normally commit to total openness but rather attempt to open-up pragmatically, responding to specific requirements by funders or taking advantage of specific opportunities. Interestingly in our cases, once scientists start opening up some part of the research project, they later usually become interested in further the opening up other dimensions and stages of the research process, sometimes through spin-offs projects.

We believe our analytical framework could be informative for researchers, policy makers and practitioners as a guide for characterizing open science experiences and also, helping to identify specific aspects of open science practices that could be opened-up further for specific targeted outcomes.

\section{Acknowledgements}

The funding for this work has been provided through the Open \& Collaborative Science in Development Network (OCSDNet) research project, supported by Canada's International Development Research Centre and the UK Government's Department for International Development. Find out more at www.ocsdnet.org and by the Interdisciplinary Centre for Studies on Science, Technology and Innovation (CIECTI) from the Ministry of Science, Technology and Productive Innovation of Argentina. In addition we would like to thank all our interviewees for their time and ideas shared with us in several opportunities and Sol Sebastian for her excellent research assistance.

\section{References}

[1] J.P. Alperin, The Public Impact of Latin American's Approach to Open Access, Stanford University, 2015.

[2] C. Årdal and J.-A. Røttingen, Open source drug discovery in practice: A case study, PLoS Neglected Tropical Diseases 6(9) (2012), e1827. doi:10.1371/journal.pntd.0001827.

[3] S.R. Arnstein, A ladder of citizen participation, Journal of the American Institute of Planners 35(4) (1969), $216-224$. doi:10.1080/01944366908977225. 
[4] B. Aronson, Improving online access to medical information for low-income countries, New England Journal of Medicine 350(10) (2004), 966-968. doi:10.1056/NEJMp048009.

[5] V. Arza and M. Fressoli, Benefits of open science: An analytical framework illustrated with case study evidence from Argentina, in: Expanding Perspectives on Open Science: Communities, Cultures and Diversity in Concepts and Practice, L. Chan and F. Loizides, eds, 2017, Ebook available at http://ebooks.iospress.nl/book/expanding-perspectiveson-open-science-communities-cultures-and-diversity-in-concepts-and-practices-proceedings-of-the-21st-internationalconference-on-electron.

[6] T. Baden, A.M. Chagas, G. Gage and T. Marzullo, Open labware: 3-D printing your own lab equipment, PLoS Biology (2015). doi:10.1371/journal.pbio.1002175.

[7] M.W. Bauer, The evolution of public understanding of science - discourse and comparative evidence, Science Technology \& Society 14(2) (2009), 221-240. doi:10.1177/097172180901400202.

[8] J. Ben-David, Roles and innovations in medicine, American Journal of Sociology (1960), 557-568. doi:10.1086/222786.

[9] Y. Benkler, The Wealth of Networks: How Social Production Transforms Markets and Freedom, Yale University Press, 2006.

[10] Y. Benkler and H. Nissenbaum, Commons-based peer production and virtue, Journal of Political Philosophy 14(4) (2006), 394-419. doi:10.1111/j.1467-9760.2006.00235.x.

[11] A. Bhardwaj, V. Scaria, G.P.S. Raghava, A.M. Lynn, N. Chandra, S. Banerjee, M.V. Raghunandanan, V. Pandey, B. Taneja, J. Yadav et al., Open source drug discovery - a new paradigm of collaborative research in tuberculosis drug development, Tuberculosis 91(5) (2011), 479-486.

[12] W. Bijker, Of Bicycles, Bakelites, and Bulbs. Toward a Theory of Sociotechnical Change, The MIT Press, Cambridge, MA, 1997.

[13] T.W. Burns, D.J. O’Connor and S.M. Stocklmayer, Science communication: A contemporary definition, Public Understanding of Science 12(2) (2003), 183-202. doi:10.1177/09636625030122004.

[14] C.L. Catlin-Groves, The citizen science landscape: From volunteers to citizen sensors and beyond, International Journal of Zoology 2012 (2012), 1-14. doi:10.1155/2012/349630.

[15] J. Cribb and T. Sari, Open Science: Sharing Knowledge in the Global Century, CSIRO Publishing, Collingwood, 2010.

[16] P. Dasgupta and P.A. David, Toward a new economics of science, Research Policy 23(5) (1994), 487-521. doi:10.1016/ 0048-7333(94)01002-1.

[17] P.A. David, The economic logic of open science and the balance between private property rights and the public domain in scientific data and information: A primer, in: The Role of the Public Domain in Scientific and Technical Data and Information, J.M. Esanu and P.F. Uhlir, eds, National Academies Press, 2003.

[18] P.A. David, Towards a cyberinfrastructure for enhanced scientific collaboration: Providing its "soft" foundations may be the hardest part, Social Science Research Network Working Paper Series 4 (2009), 1-24.

[19] A. Delfanti, Users and peers. From citizen science to P2p science, Journal of Science Communication 9(1) (2010), 1-5.

[20] European Commission, Open Innovation, Open Science, Open to the World, European Commission, Brussels, 2016.

[21] B. Fecher and S. Friesike, Open science: One term, five schools of thought, in: Opening Science, Springer, 2014.

[22] U. Felt, D. Barben, A. Irwin, P.-B. Joly, A. Rip, A. Stirling and T. Stöckelová, Science in society: Caring for our futures in turbulent times, Policy Briefing 50, 2013.

[23] M. Franco-Avellaneda, Museos, Artefactos Y Sociedad: ¿Cómo Se Configura Su Dimensión Educativa?, Universitas Humanística 76 (2013), 97-123.

[24] C. Franzoni and H. Sauermann, Crowd science: The organization of scientific research in open collaborative projects, Research Policy 43(1) (2014), 1-20.

[25] M. Fressoli and V. Arza, Negociando la apertura en ciencia abierta. Un análisis de casos ejemplares de Argentina, Revista Iberoamericana de Ciencia, Tecnología y Sociedad, 2016, in press.

[26] D. Gagliardi, D. Cox and Y. Li, Institutional inertia and barriers to the adoption of open science, in: The Transformation of University Institutional and Organizational Boundaries, E. Reale and E. Primeri, eds, Sense Publishers, 2015.

[27] J. Gregson, J.M. Brownlee, R. Playforth and N. Bimbe, The Future of Knowledge Sharing in a Digital Age: Exploring Impacts and Policy Implications for Development, The Institute of Development Studies, Sussex, Brighton, 2015.

[28] A.M. Grubb and S.M. Easterbrook, On the lack of consensus over the meaning of openness: An empirical study, PLoS One 6(8) (2011). doi:10.1371/journal.pone.0023420.

[29] J.K. Hartshorne and A. Schachner, Tracking replicability as a method of postpublication open evaluation, Frontiers in Computational Neuroscience (2012).

[30] M. Hu, K. Schultz, J. Sheu and D. Tschopp, The Innovation Gap in Pharmaceutical Drug Discovery \& New Models for $R \& D$ Success, Kellogg School of Management, 2007.

[31] J.P.A. Ioannidis, Evidence-based medicine has been hijacked: A report to David Sackett, Journal of Clinical Epidemiology 73 (2016), 82-86. doi:10.1016/j.jclinepi.2016.02.012.

[32] L.B. Jeppensen and K. Lakhani, Marginality and problem-solving effectiveness in broadcast search, Organization Science 21(5) (2010), 1016-1033. doi:10.1287/orsc.1090.0491. 
[33] P. Kreimer, Ciencia Y Periferia: Una Lectura Sociológica, in: La Historia De La Ciencia En El Siglo Xx Manantial, M. M. (Ed.), eds, Buenos Aires, 1998.

[34] C. Lagoze, Ebird: Curating citizen science data for use by diverse communities, International Journal of Digital Curation 9(1) (2014), 71-82.

[35] B.-Å. Lundvall, National Systems of Innovation: Toward a Theory of Innovation and Interactive Learning, Pinter Publishers; Distributed Exclusively in the USA and Canada by St. Martin's Press, London; New York, 1992.

[36] R. Mansell, Employing digital crowdsourced information resources: Managing the emerging information commons, International Journal of the Commons 7(2) (2013).

[37] B. Martin, Strategies for alternative science, in: S. Fricklel and K. Moore, eds, University of Wisconsin Press, Madison, 2005.

[38] H. Masum and R. Harris, Open source for negleted diseases: Challenges and opportunities, Center for Global Health R\&D Policy Assessment, 2011.

[39] R. Mazzoleni and R.R. Nelson, Public research institutions and economic catch-up, Research Policy 36(10) (2007), 15121528.

[40] R.K. Merton, Priorities in scientific discovery. A chapter in sociology of science, American Sociological Review 22(6) (1957), 635-659.

[41] J. Molloy, Open training for open science, 2014, available at http://science.okfn.org/2014/12/21/open-training-for-openscience/.

[42] J.C. Molloy, The open knowledge foundation: Open data means better science, PLoS Biology 9(12) (2011), e1001195. doi:10.1371/journal.pbio.1001195.

[43] D.C. Mowery, The practice of technology policy, in: Handbook of the Economics of Innovation and Technological Change, P. Stoneman, ed., Blackwell, Oxford, 1995.

[44] R.R. Nelson, The market economy, and the scientific commons, Research Policy 33(3) (2004), 455-471. doi:10.1016/j. respol.2003.09.008.

[45] M. Nielsen, Reinventing Discovery: The New Era of Networked Science, Princeton University Press, New Jersey, 2012.

[46] OECD, Science, Technology, and Innovation for the 21st Century: Meeting of the Oecd Committee for Scientific and Technological Policy at Ministerial Level, 2004.

[47] OECD, Making open science a reality, OECD Science, Technology and Industry Policy Papers, n. 25, OECD Publishing, Paris, 2015.

[48] RIN/NESTA, Open to All? Case Studies of Openness in Research. Research Information Network (RIN) and National Endowment for Science, Technology and the Arts (NESTA), London, 2010.

[49] M.N. Robertson, P.M. Ylioja, A.E. Williamson, M. Woelfle, M. Robins, K.A. Badiola, P. Willis, P. Olliaro, T.N.C. Wells and M.H. Todd, Open source drug discovery - limited tutorial, Parasitology 141(01) (2014), 148-157. doi:10.1017/ S0031182013001121.

[50] C.M. Schweick, Free/open source software as a framework for establishing commons in science, in: Understanding Knowledge as a Commons, C.H.E. Ostrom, ed., The MIT Press, Boston, Massachusetts, 2011.

[51] J. Silvertown, A new dawn for citizen science, Trends in Ecology \& Evolution 24(9) (2009), 467-471. doi:10.1016/j.tree. 2009.03.017.

[52] B. Stadelmann, R. Rufener, D. Aeschbacher, M. Spiliotis, B. Gottstein and A. Hemphill, Screening of the open source malaria box reveals an early lead compound for the treatment of alveolar echinococcosis, PLoS Neglected Tropical Diseases 10(3) (2016). doi:10.1371/journal.pntd.0004535.

[53] V. Stodden, Open science: Policy implications for the evolving phenomenon of user-led scientific innovation, Journal of Science Communication 9(1) (2010), 1-8.

[54] P. Suber, The taxpayer argument for open access, SPARC Open Access Newsletter (2003).

[55] J. Surowiecki, The Wisdom of Crowds: Why the Many Are Smarter than the Few and How Collective Wisdom Shapes Business, Economies, Societies and Nations Little, Anchor Books, New York, 2004.

[56] UN Independent Expert Advisory Group Secretary, A World That Counts: Mobilising the Data Revolution for Sustainable Development, United Nations Independent Expert Advisory Group on a Data Revolution for Sustainable Development, United Nations, 2014.

[57] S. Weber, The Success of Open Source, Harvard University Press, 2004.

[58] T. Wells, W.C. Van Voorhis, J.H. Adams, R. Adelfio, V. Ahyong, M.H. Akabas, P. Alano, A. Alday, Y. Alemán Resto, A. Alsibaee et al., Open source drug discovery with the malaria box compound collection for neglected diseases and beyond, PLoS Pathogens 12(7) (2016). doi:10.1371/journal.ppat.1005763.

[59] A. Whyte and G. Pryor, Open science in practice: Researcher perspectives and participation, International Journal of Digital Curation 6(1) (2011), 199-213. doi:10.2218/ijdc.v6i1.182.

[60] A. Wiggins and K. Crowston, From Conservation to Crowdsourcing: A Typology of Citizen Science, Hawai, 2011.

[61] World Bank, Open Data for Sustainable Development, World Bank, 2015. 